\title{
Synchronous PV Support to an Isolated Power System
}

\author{
Daniel Remon* ${ }^{* \ddagger}$, Antoni M. Cantarellas* ${ }^{*}$, Mohamed Atef Abbas Elsaharty ${ }^{\ddagger}$, \\ Cosmin Koch-Ciobotaru*, Pedro Rodriguez ${ }^{* \ddagger}$ \\ *Abengoa Research, Abengoa \\ Campus Palmas Altas, Seville, Spain 41014 \\ Email: daniel.remon@abengoa.com \\ ${ }^{\ddagger}$ Department of Electrical Engineering \\ Technical University of Catalonia \\ GAIA Building, Terrassa, Barcelona, Spain 08222
}

\begin{abstract}
The increase of renewable energy sources and distributed generation may have a negative impact on power systems, specially on weak ones. Therefore, their control systems are acquiring growing importance, with new proposals that aim to contribute to the control and stability of the power system as conventional generating units.

This paper studies the effect that a PV system using a synchronous power controller may have on a small power system constituted by a diesel generator, two loads and the PV generating unit. The response of this power system to realistic disturbances is compared with the case where the PV unit is controlled to provide its maximum power production regardless of the state of the system.
\end{abstract}

Index Terms-distributed generation, grid support, power system stability, renewable energy sources.

\section{INTRODUCTION}

Renewable energy sources like PV and wind energy and distributed generation are increasing their participation in power systems and it is predicted that this trend will continue during the following years [1]. Although this is positive from the point of view of reducing $\mathrm{CO}_{2}$ emissions and developing a sustainable and efficient electrical system, it also brings some challenges for power system operators, since this type of generating units may have a negative impact on power systems.

Usually, these units are connected to the grid through power electronics and their dynamics do not match those of synchronous generators, which determine the main characteristics of classical power systems [2], [3]. One of the main impacts of this kind of generators is that they displace conventional generators, reducing the total inertia of the system, which may result in larger frequency variations and a more unstable behavior. Moreover, they cannot normally be dispatched as conventional generators based on fossil fuels or hydro power and the system must compensate their variability.

This work has been partially supported by the Spanish Ministry of Science and Innovation under the project ENE2014-60228-R.

Any opinions, findings and conclusions or recommendations expressed in this material are those of the authors and do not necessarily reflect those of the host institutions or funders.
This issue is especially important in weak power systems that have a reduced number of generators and are poorly interconnected to a larger system. These generators are traditionally controlled to maintain the supply and demand balance. However, when renewable sources are connected to such a system, displacing some of these conventional generators, the total inertia of the system can be very low. Additionally, they introduce new sources of variability and, if they are not properly managed, they may reduce the amount of resources that can be employed to control the system and ensure a safe and stable operation.

Several control systems for power converters have been proposed during the last years to mitigate the impact that an unresponsive generating unit might have on the power system. In addition to including the conventional droop response to participate in primary regulation, several authors have proposed different ways of emulating inertia or the dynamics of synchronous machines [4]-[10]. Among them, the synchronous power controller (SPC) introduced in [9] is able to emulate inertia and interact synchronously with the power system like synchronous machines. However, the SPC does not mimic the complete dynamics of these machines, avoiding their weak damping and the complexity of magnetization circuits. Additionally, it presents other advantages like allowing the definition and modification of the inertia and damping values, using a virtual admittance, being adaptable to different harmonics and unbalanced systems and avoiding the need for synchronization systems like phase-locked loops or frequencylocked loops, whose dynamics influence the response of the controller [11], [12].

Taking into account the impact that renewable generators interfaced by power electronics may have on weak power systems depending on their control, this paper analyzes the performance of a small power system constituted by a diesel generator, two loads and a PV generating unit in two scenarios. In the first one, the PV unit behaves as an unresponsive power injection, whereas in the second one it responds synchronously through the SPC. In both scenarios, the response to different 


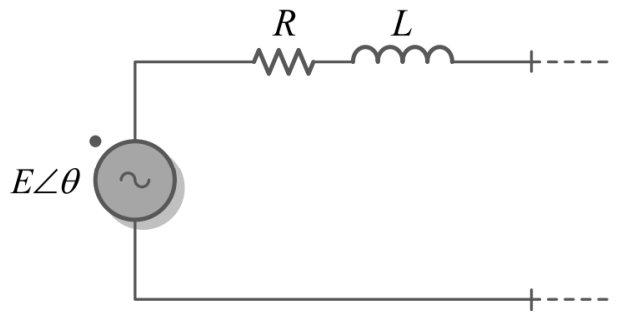

Fig. 1. Simplified electrical equivalent of a synchronous machine.

disturbances caused by the load and the solar resource is studied.

The rest of the paper is organized as follows. Section II explains the control system of the PV unit using the SPC and section III defines the power system to be analyzed. Then, section IV presents the analysis of the response of the power system with and without a responsive control of the PV unit. Finally, section $\mathrm{V}$ summarizes the main conclusions of the work.

\section{The Synchronous POWFr CONTROLI.ter}

The PV unit analyzed in this paper is connected to the AC grid through a voltage source converter. The control system of the converter consists on a modulation, a current controller and a power controller. Additionally, a DC link voltage controller and a resource controller ensure that the DC link voltage keeps its rated value by modifying the power production of a PV source working under curtailment or a small storage system in order to provide support to the grid.

The internal loops allow identifying the converter and its grid filter with a controlled current source, whereas the power controller generates the reference for this current source and determines its main dynamics. In this case, the synchronous power controller protected by [8] and presented in [9], [10] is used.

The SPC embeds and improves the main features of a synchronous machine in the response of the converter. It reproduces the simplified model of a synchronous machine shown in Fig. 1, where it is seen as a voltage source behind an impedance. In this way, the converter is able to form a grid and to contribute to the stability of the system during disturbances. Moreover, the angle of the internal voltage source behaves as in the case of a synchronous machine, with its dynamics dictated by a swing equation with damping. A conceptual diagram of the main SPC blocks is given by Fig. 2 .

\section{A. Reactive power controller}

The magnitude $E$ of the internal voltage source is determined by the reactive power controller, avoiding the complexity of magnetization circuits of synchronous machines. This controller regulates the injection of reactive power or the voltage at a bus near the converter. It can be constituted by a proportional, a proportional-integral, or a more complex controller including filters or lead-lag compensators.

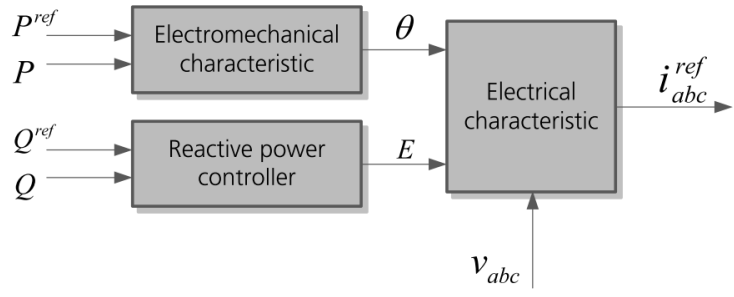

Fig. 2. Constituting blocks of the synchronous power controller.

In this case, a simple proportional-integral controller acting on the reactive power error is employed. Additionally, its reactive power reference can be modified by a higher level controller to control the voltage at a given point.

\section{B. Electromechanical Characteristic}

The electromechanical characteristic of the SPC reproduces the swing equation of a rotating mass with damping, given by (1), where $\omega$ is the rotor speed, $J$ is its moment of inertia, $D$ is the damping coefficient of the machine and $P_{\text {mech }}$ and $P_{\text {elec }}$ are respectively the input and output power. However, in the case of a converter, the moment of inertia and the damping coefficient can be modified in real time in order to adapt to power system conditions, and it is possible to obtain highly damped responses.

$$
J \frac{d \omega}{d t}=\frac{P_{m e c h}-P_{\text {elec }}}{\omega}-D \Delta \omega
$$

In a converter, the input power in (1) depends on the active power reference, whereas the output power given by the model in Fig. 1 depends on the internal machine voltage, the grid voltage, the total impedance and the load angle. In small signal, the variation of the output power as a result of a variation of the load angle can be expressed by (2). In this equation, $T_{P}$ depends on the operating point, as given by (3), and its value can be updated online to adapt to changing system conditions.

$$
\begin{gathered}
\Delta P_{\text {out }}=T_{P} \Delta \delta \\
T_{P}=\frac{\partial P_{\text {out }}}{\partial \delta}\left(\delta_{0}\right)
\end{gathered}
$$

Therefore, in small signal, (1) can be written as (4), where $\omega_{g}$ is the rated rotor speed and $\zeta$ is the desired damping coefficient. Considering the whole active power loop of Fig. 3 , the closed-loop transfer function is given by (5).

$$
\begin{gathered}
\frac{\Delta \omega}{\Delta P}(s)=\frac{1}{J \omega_{g}} \frac{1}{s+2 \zeta \sqrt{\frac{T_{P}}{J \omega_{g}}}} \\
\frac{\Delta P_{\text {out }}}{\Delta P_{\text {ref }}}(s)=\frac{\frac{T_{P}}{J \omega_{g}}}{s^{2}+2 \zeta \sqrt{\frac{T_{P}}{J \omega_{g}}} s+\frac{T_{P}}{J \omega_{g}}}
\end{gathered}
$$




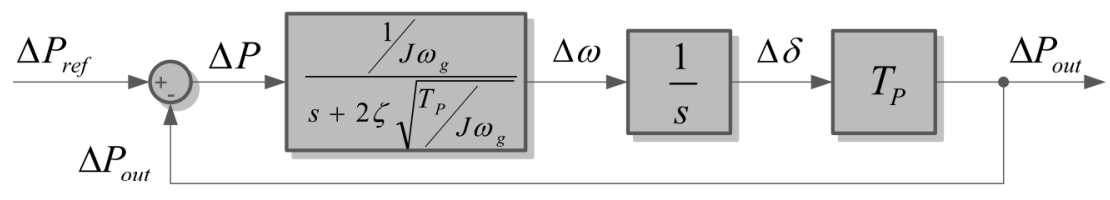

Fig. 3. Active power loop with the synchronous power controller emulating inertia $J$ with damping coefficient $\zeta$.

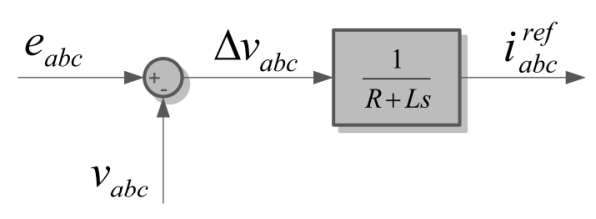

Fig. 4. Current reference calculation from the voltage drop through the virtual admittance.

\section{Electrical Characteristic}

Taking the values of the internal voltage magnitude and angle respectively provided by the reactive power controller and the electromechanical characteristic blocks, the electrical characteristic block determines the current that must be injected by the converter to reproduce the model in Fig. 1. In this block, the three-phase internal voltage is compared with the grid voltage in order to determine the voltage drop through the virtual impedance. This voltage drop induces a current that is given by (6). In small signal, the current of each phase can be obtained from the corresponding voltage drop as in (7), which results in the control diagram of Fig. 4.

$$
\begin{gathered}
\frac{d i}{d t}=\frac{1}{L}(\Delta v-R i) \\
\frac{\Delta i}{\Delta v}(s)=\frac{1}{R+L s}
\end{gathered}
$$

From Fig. 4, it is possible to see that the virtual impedance is in fact applied as a virtual admittance that filters the voltage drop. This results in a more stable control loop and avoids problems of derivative terms when a virtual impedance is applied to a current signal to calculate the voltage reference to be followed by the converter.

\section{Higher-level controllers}

The active and reactive power references are modified by higher-level controllers that regulate the frequency and the voltage.

The contribution of the converter to frequency regulation is defined by a droop response that modifies the active power reference. The converter reference increases when the system frequency decreases and vice versa, as defined by equation (8). The proportional nature of this controller allows connecting more generating units that contribute to frequency regulation in the same way. It is also compatible with a higher-level secondary controller or a single isochronous governor in one

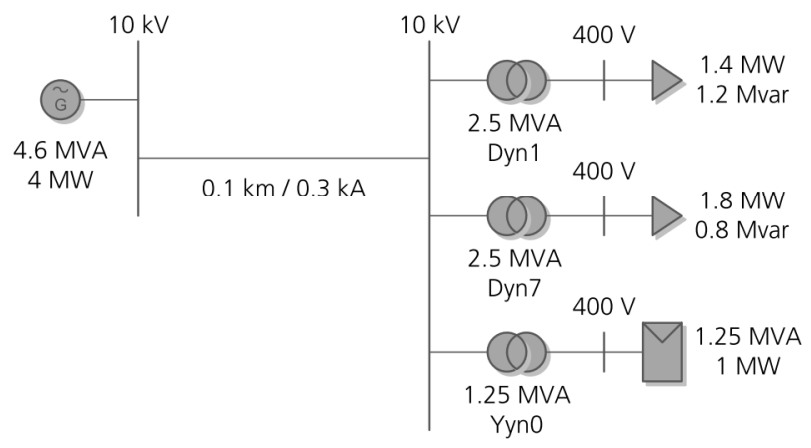

Fig. 5. Single-line diagram of the test system.

of the participating units, which would maintain the frequency at its rated value.

$$
\Delta P_{\text {droop }}=\frac{f_{\text {ref }}-f}{R_{f}}
$$

Similarly, the voltage at the converter terminals modifies the converter reactive power reference following (9). This proportional relation also allows several units to control the voltage at the same bus or in a close area, avoiding that their controllers fight among them.

$$
\Delta Q_{\text {droop }}=\frac{V_{r e f}-V}{R_{v}}
$$

\section{Test SySTEM}

In order to demonstrate the contribution of the SPC to power system stability, its performance is tested in a weak system. This system is mainly powered by a $4 \mathrm{MW}$ diesel generator at medium voltage and feeds several low voltage loads that are represented by two equivalent loads with a constant impedance model. The consumption of these loads is $1.4 \mathrm{MW}$ and $1.2 \mathrm{Mvar}$ in the case of load 1 and $1.8 \mathrm{MW}$ and 0.8 Mvar in the case of load 2. Additionally, a $1 \mathrm{MW}$ PV system contributes to power generation in this system during certain hours everyday. The complete system is modeled in DIgSILENT PowerFactory and its single-line diagram is shown in Fig. 5.

The diesel generator is the main element in the control of the power system. Its inertia time constant is $H=2.39 \mathrm{~s}$ and it is equipped with an isochronous governor that regulates the system frequency. This means it introduces an integral action in order to keep the frequency at its rated value. It also receives an input signal from an automatic voltage regulator (AVR) to control its terminal voltage. 
For the PV system, two operation modes are considered. The first is a traditional one, with the PV system programmed to inject a constant amount of power equal to the maximum possible production. In the second one, the PV system is controlled by the SPC in order to support the system. It emulates an inertia of $5 \mathrm{~s}$ and contributes to frequency regulation with a droop response with constant $R_{f}=0.05 \mathrm{pu}$. It also modifies its reactive power injection in order to control the voltage at the common bar to which the loads and the PV system are connected, also with a droop constant $R_{v}=0.05 \mathrm{pu}$. Its damping coefficient is set to $\zeta=0.8$.

\section{RESULTS}

The performance of the power system is tested under two different disturbances. The first one consists on a step increase in load 1, whereas the second disturbance is due to the solar resource, with a fast decrease in radiation which is transmitted to the power generation of the PV system. In both cases, the response of the system with PV contributing to its control is compared to the response of the system when the PV system behaves only as a power injection following the solar resource.

When emulating inertia, the PV system reproduces the effect of extracting kinetic energy of the rotor and injecting it into the grid that takes place naturally within a synchronous machine. In this case, the converter requires the support of a storage system like a supercapacitor connected to its DC link.

\section{A. Load Increase}

In the first study case, the consumption of load 1 suddenly increases from $1.4 \mathrm{MW}$ and $1.2 \mathrm{Mvar}$ to $1.96 \mathrm{MW}$ and $1.38 \mathrm{Mvar}$ at $t=0 \mathrm{~s}$ due to the connection of additional loads. During the short period of time that the system is affected by the load increase, the input power of the PV system can be considered constant.

This increase has an effect on the power production and the consumption of both loads, as can be seen in Fig. 6. This figure allows comparing the response under both types of control of the PV unit, with dashed lines for the conventional control and solid lines for the synchronous one.

When the PV system is controlled by the SPC, the initial effort is shared between the diesel generator and the PV converter, which contributes to reduce the speed with which the generator is loaded, providing a significant amount of power during the first two seconds and contributing with an increase of power until $t=10 \mathrm{~s}$, utilizing the energy stored in the DC link supercapacitor. This contribution is reflected in the power consumed by both loads, which reaches a steady state almost immediately after load 1 is increased, whereas it suffers transients of approximately $3 \mathrm{~s}$ without the contribution of the PV unit.

In Fig. 7, the evolution of the voltages at the buses where the generator, the loads and the PV system are connected is shown. The support of the PV system allows reducing the $3 \mathrm{~s}$ transients that take place when the generator alone is controlling the power system state.

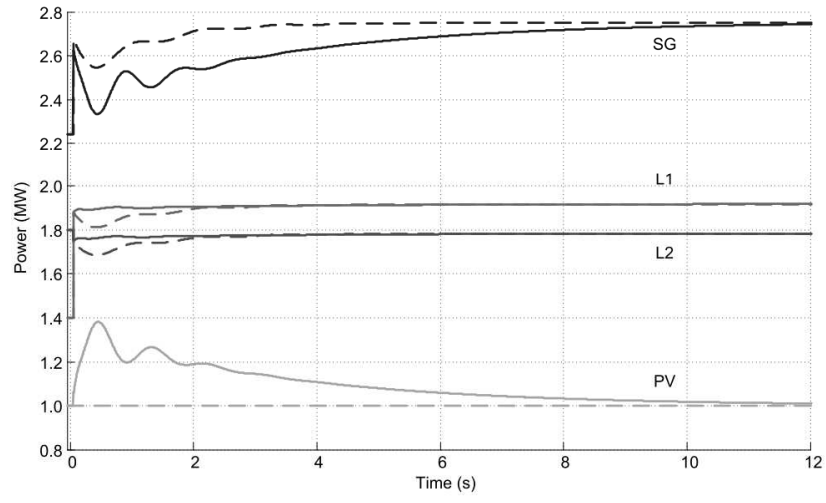

Fig. 6. Active power after a step increase in load 1 consumption: Generated by synchronous generator (SG) and PV unit (PV) and consumed by load 1 (L1) and load 2 (L2). Dashed lines: constant PV injection. Solid lines: synchronous PV.

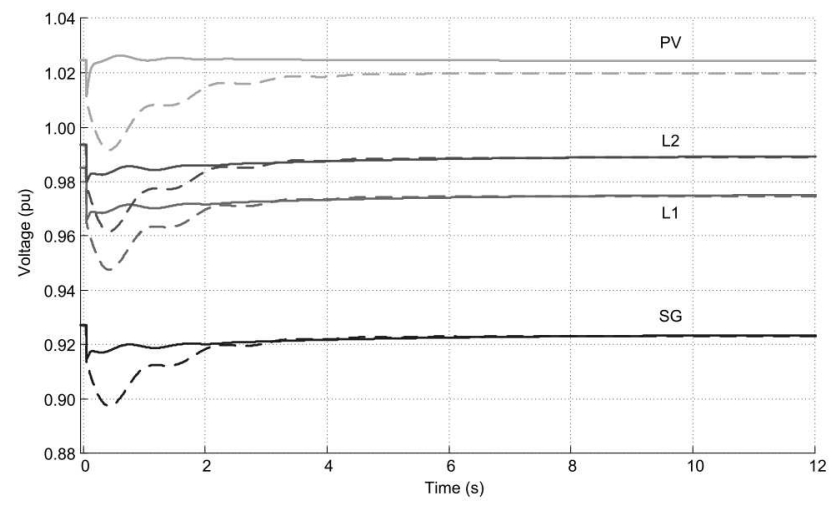

Fig. 7. Bus voltage after a step increase in load 1 consumption: Synchronous generator (SG), PV unit (PV), load 1 (L1) and load 2 (L2). Dashed lines: constant PV injection. Solid lines: synchronous PV.

In this case, the voltage of the generator reaches values below $0.9 \mathrm{pu}$, which may be harmful for the machine, and in some cases may even cause its disconnection and the subsequent collapse of the system. When the PV unit behaves as a synchronous generator, the voltage deviation at the generator terminals is reduced and the minimum value is not far from its final steady state value, reducing the probability of an important failure.

Something similar occurs with the load voltages. In particular, the voltage of load 1 goes below $0.95 \mathrm{pu}$ without a responsive PV controller, which may also lead to the disconnection of loads and create a problem for customers, whereas it is kept safely over $0.96 \mathrm{pu}$ when the PV system supports the system.

Finally, the frequency of the system, given by the speed of the diesel generator rotor and the internal SPC frequency, is shown in Fig. 8. In this figure it is possible to see how the internal frequency of the SPC follows the frequency of the diesel generator because the controller is working in synchronism with the power system. Furthermore, Fig. 8 allows observing the difference in the maximum deviation 


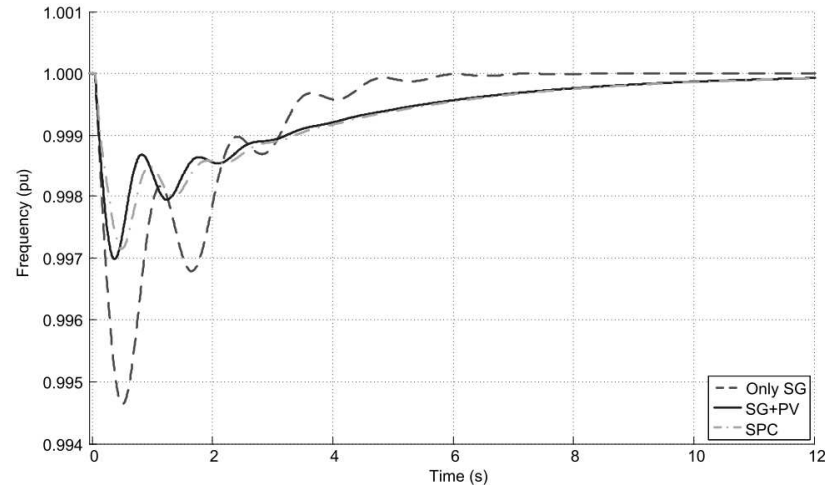

Fig. 8. Frequency after a step increase in load 1 consumption. Dashed line constant PV injection. Solid line: synchronous PV. Dash-dotted line: SPC estimation in the synchronous PV case.

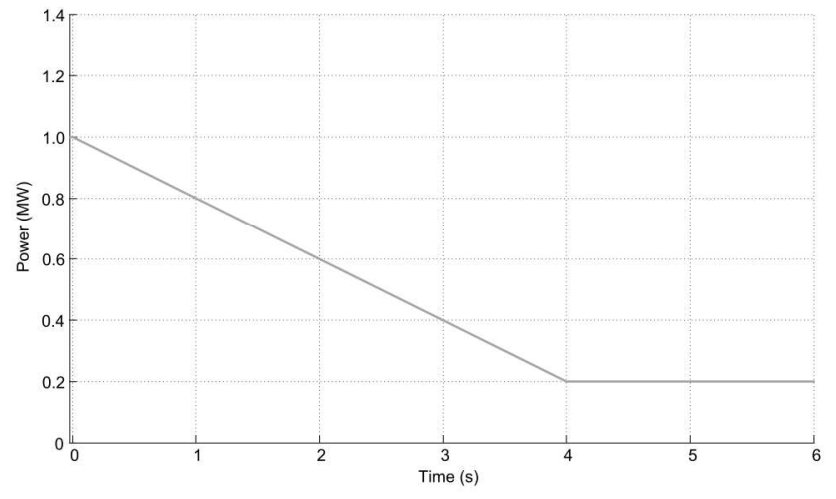

Fig. 9. DC active power available at the PV system due to a ramp decrease in solar radiation

of the generator speed thanks to the contribution of the PV system.

Moreover, the high damping coefficient of the SPC control loop allows reducing the frequency oscillations during the whole transient and its additional inertia makes transients slower, limiting both maximum deviations and the speed with which the generator is forced to act. Obviously, the increase in the total inertia also causes the system frequency to reach a steady state later, but the frequency deviation is smaller in the case without PV contribution only when it is back within a $0.1 \%$ band and the system has already stabilized.

\section{B. Solar Resource Decrease}

In the second study case, the power produced by the PV system decreases due to a cloud. Initially at $1 \mathrm{MW}$, it linearly decreases down to $0.2 \mathrm{MW}$ in $4 \mathrm{~s}$ and then this low radiation level is maintained. The corresponding active power profile is shown in Fig. 9.

When the PV unit is controlled by the SPC, it uses the energy storage to smooth the response of the PV system and reduce the effects of this variation. Moreover, the output power decrease is not directly dictated by the resource variation, but given by the synchronous interaction between the SPC and the

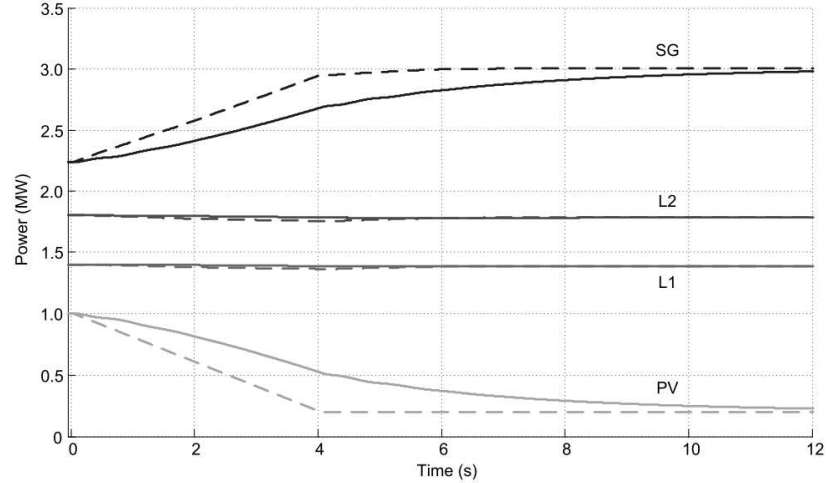

Fig. 10. Active power during a ramp decrease in solar radiation: Generated by synchronous generator (SG) and PV unit (PV) and consumed by load 1 (L1) and load 2 (L2). Dashed lines: constant PV injection. Solid lines: synchronous PV.

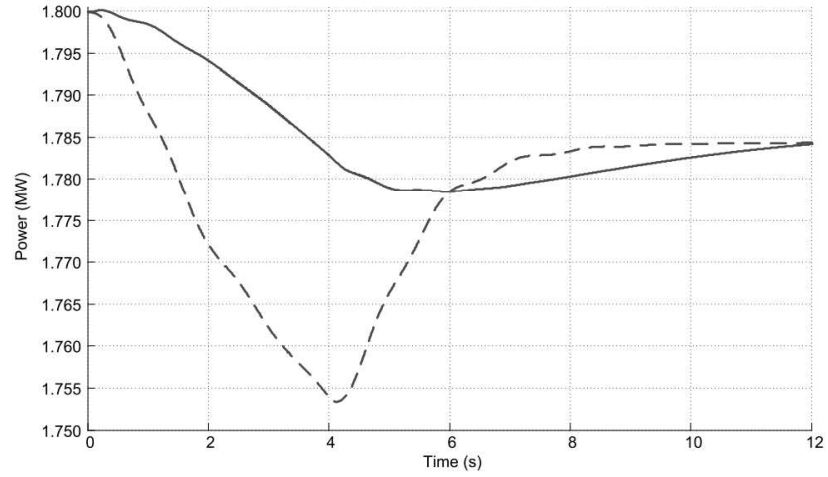

Fig. 11. Active power consumed by load 2 during a ramp decrease in solar radiation. Dashed line: constant PV injection. Solid line: synchronous PV.

power system.

Fig. 10 is a comparison of the active power injected by the synchronous generator and the PV unit and consumed by both loads, for both PV control modes. It shows how the contribution of the PV unit relieves the synchronous generator, allowing it to reduce the speed with which its load is increased in order to compensate the reduction in the PV power production.

Thanks to the fast response of the diesel generator, the power consumption of both loads in Fig. 10 appears to be almost unaffected even when the PV unit does not contribute to the control. Nevertheless, Fig. 11 indicates that the contribution of the PV system is still able to improve the power quality at the loads by smoothing the power transient.

The effect on the voltages is more evident, as shown in Fig. 12. Without support from the PV system, the voltages reach a minimum value around $t=4 \mathrm{~s}$ and then rise again until $t=6 \mathrm{~s}$, when they reach the final steady state value. Contrary to this, with a PV system governed by the SPC, the voltage is reduced linearly to the final value in $6 \mathrm{~s}$. Therefore, the variation sensed by the loads and also the PV system is less evident. 


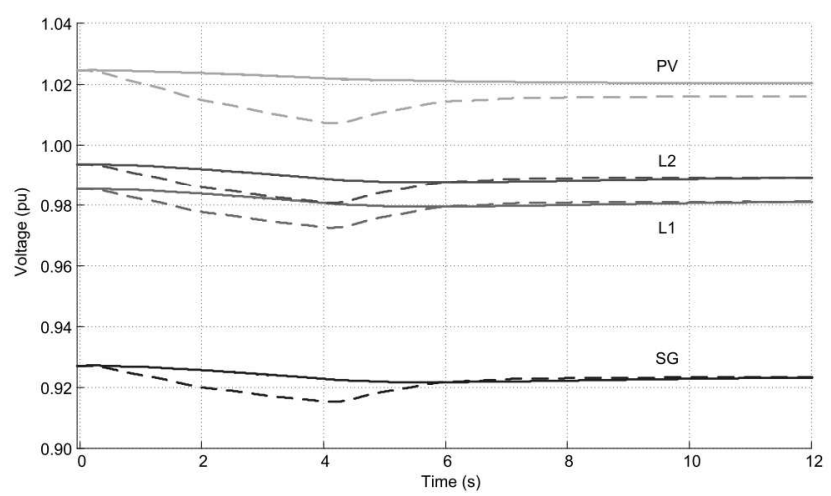

Fig. 12. Bus voltage during a ramp decrease in solar radiation: Synchronous generator (SG), PV unit (PV), load 1 (L1) and load 2 (L2). Dashed lines: constant PV injection. Solid lines: synchronous PV.

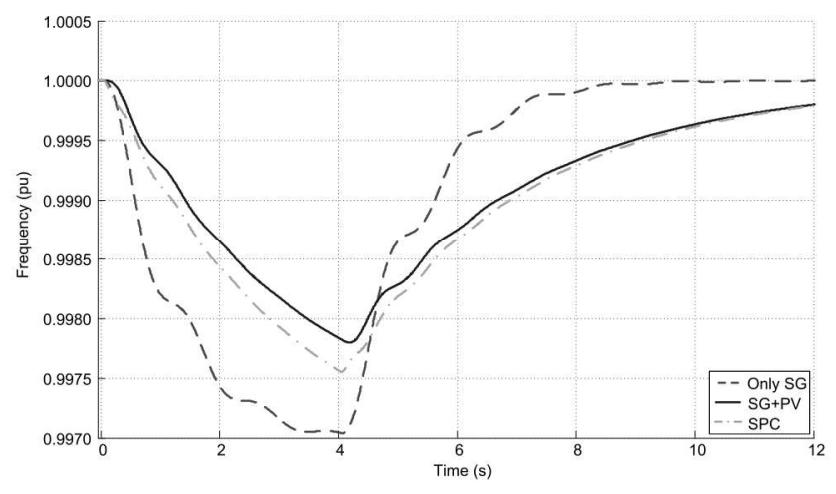

Fig. 13. Frequency during a ramp decrease in solar radiation. Dashed line constant PV injection. Solid line: synchronous PV. Dash-dotted line: SPC estimation in the synchronous PV case.

Finally, in Fig. 13, it is possible to see the effect of this active power variation on the frequency of the system. Both with and without the contribution of the PV unit, the system frequency decelerates while the PV power decreases, reaching its minimum value at $t=4 \mathrm{~s}$, and starts recovering when the solar radiation becomes constant.

Nonetheless, there are differences depending on how the PV system is controlled. The additional inertia provided by the PV system with the SPC reduces the maximum frequency deviation and at the same time makes the response slower. Furthermore, the highly damped response of the SPC has an important effect on the frequency oscillations which fade after two seconds when this controller is employed.

\section{CONCLUSION}

This paper analyzes the ability of a PV generation system using the synchronous power controller to contribute to the stability of the power system to which the PV unit is connected. The SPC allows a power converter to reproduce the main characteristics of a synchronous machine. First, it constitutes a voltage source able to contribute to power system stability and to form a grid. Second, it responds as described by the swing equation, which makes the converter work in synchronism and automatically respond to frequency variations. Furthermore, it improves the dynamics of synchronous machines because it avoids the complexity of excitation systems and has flexibility to define different values of inertia, damping and impedance.

The contribution of such a system is more evident in a weak power system where the PV unit has a significant impact. Therefore, in this paper a small system constituted by a diesel generator, two loads and the PV generating unit has been studied. Its performance has been analyzed and compared in two different cases. In the first one, the PV system simply follows the maximum power production of the resource and does not support the system. In the second one, it is controlled by the SPC and therefore contributes to power system control and stability like a synchronous machine.

For this comparison, the paper presents the response of the complete power system under two disturbances that may take place in such a system: a sudden increase of load and a fast reduction of PV production due to a cloud. In both cases, the synchronous response of the PV controlled by the SPC allows reducing the power transients at the loads, the speed with which the diesel generator must react and the effects on the voltage and the frequency of the system, working within safer operation limits and resulting in better power quality.

\section{REFERENCES}

[1] International Energy Agency, World Energy Outlook 2014. Paris: OECD/IEA, 2014.

[2] P. Kundur, Power System Stability and Control. New York: McGrawHill, 1994.

[3] J. Machowski, J. W. Bialek, and J. R. Bumby, Power System Dynamics: Stability and Control. Chichester: John Wiley \& Sons, 2008.

[4] N. Miller, R. W. Delmerico, K. Kuruvilla, and M. Shao, "Frequency responsive controls for wind plants in grids with wind high penetration," in Power and Energy Society General Meeting, 2012 IEEE, July 2012, pp. $1-7$.

[5] H. Bevrani, T. Ise, and Y. Miura, "Virtual synchronous generators: A survey and new perspectives," Electrical Power and Energy Systems, vol. 54, pp. 244-254, 2014.

[6] L. Zhang, L. Harnefors, and H.-P. Nee, "Power-synchronization control of grid-connected voltage-source converters," Power Systems, IEEE Transactions on, vol. 25, no. 2, pp. 809-820, May 2010.

[7] Q.-C. Zhong, P.-L. Nguyen, Z. Ma, and W. Sheng, "Self-synchronized synchronverters: Inverters without a dedicated synchronization unit," Power Electronics, IEEE Transactions on, vol. 29, no. 2, pp. 617-630, Feb 2014.

[8] P. Rodriguez Cortes, J. I. Candela Garcia, J. Rocabert Delgado, and R. Teodorescu, "Synchronous power controller for a generating system based on static power converters," International Patent WO 2012/117 131 A1, Sep. 7, 2012.

[9] P. Rodriguez, I. Candela, and A. Luna, "Control of PV generation systems using the synchronous power controller," in Energy Conversion Congress and Exposition (ECCE), 2013 IEEE, Sept 2013, pp. 993-998.

[10] D. Remon, A. M. Cantarellas, E. Rakhshani, I. Candela, and P. Rodriguez, "An active power self-synchronizing controller for gridconnected converters emulating inertia," in Renewable Energy Research and Applications (ICRERA), 2014 International Conference on, Oct 2014 , pp. $1-5$.

[11] F. Blaabjerg, R. Teodorescu, M. Liserre, and A. Timbus, "Overview of control and grid synchronization for distributed power generation systems," Industrial Electronics, IEEE Transactions on, vol. 53, no. 5, pp. 1398-1409, Oct 2006.

[12] R. Teodorescu, M. Liserre, and P. Rodriguez, Grid Converters for Photovoltaic and Wind Power Systems. Chichester: Wiley, 2011. 